\title{
GENERALIZED SUPER-SOLUTIONS OF PARABOLIC EQUATIONS
}

BY

\author{
NEIL A. EKLUND
}

\begin{abstract}
Let $L$ be a linear, second order parabolic operator in divergence form and let $Q$ be a bounded cylindrical domain in $E^{n+1}$. Supersolutions of $L u=0$ are defined and generalized to three equivalent forms. Generalized super-solutions are shown to satisfy a minimum principle and form a lattice.
\end{abstract}

Consider the parabolic operators $L$ of the form

$$
L u=u_{t}-\left\{a_{i j}(x, t) u_{, i}+d_{j}(x, t) u\right\}_{, j}-b_{j}(x, t) u_{, j}-c(x, t) u
$$

where summation occurs over the repeated indices $i, j$. The assumptions made on the coefficient functions can be found in reference [7]. The numbering of definitions, theorems, and references will continue sequentially from those in reference [7].

In this paper super-solutions of $L u=0$ are introduced assuming weak differentiability properties; a minimum principle is proved for such functions. Then the class of super-solutions is generalized to functions which may not possess weak derivatives. Three definitions of such functions are given and they are shown to be equivalent. Then some properties of these functions are obtained.

III. Minimum principle. The classical minimum principle for superparabolic operators $L$ on cylinders $Q$ in $E^{n+1}$ states that $u \in C^{2}(Q) \cap C(\bar{Q})$, $L u \geqslant 0$ in $Q$, and $u \geqslant 0$ on $\partial_{p} Q=\{\Omega \times(0)\} \cup\{\partial \Omega \times[0, T]\}$, the parabolic boundary of $Q$, imply that $u \geqslant 0$ on $Q$. The same result can be obtained for weak parabolic operators $L$ provided the proper interpretation of these assumptions is made.

DEFinition 2. $u=u(x, t)$ is called a super-solution of $L u=0$ in $Q$ $\left(u \in S_{Q}\right)$ if $u \in L^{2}\left[0, T ; H^{1,2}(\Omega)\right]$ and, for all $v \in C_{0}^{1}\left(Q^{i}\right)$ with $v \geqslant 0$.

$$
\iint_{Q}\left[a_{i j} u_{r_{i}} v_{, j}+d_{j} v_{j j} u-b_{j} u_{, j} v-c u v-u v_{t}\right] d x d t \geqslant 0 .
$$

Presented to the Society, January 17, 1974; received by the editors October 1, 1973. 35 RO5.

AMS (MOS) subject classifications (1970). Primary $35 \mathrm{K20}, 31 \mathrm{B05}$; Secondary

Key words and phrases. Parabolic PDE, super-solutions, potential theory. 
The notion of $u \geqslant 0$ on $\partial_{p} Q$ must be introduced since $u$ is defined only almost everywhere on $Q$.

Definition 3. $u \in L^{2}\left[0, T ; H^{1,2}(\Omega)\right]$ is nonnegative on $\partial_{p} Q$ if there is a sequence of functio:ss $\left\{u^{k}(x, t)\right\} \subset C(\bar{Q})$ with $u^{k}$ lipshitz continuous in $x$ on $\bar{\Omega}$ uniformly in $t, u^{k}>0$ on $\partial_{p} Q$, and $u^{k} \rightarrow u$ in $L^{2}\left[0, T ; H^{1,2}(\Omega)\right]$.

Theorem 3. (MINIMUM PRINCIPLE). Let $u \in S_{Q}$ with $u \geqslant 0$ on $\partial_{p} Q$ and having initial values $u_{0} \in L^{2}(\Omega)$. Then $u \geqslant 0$ a.e. in $Q$.

Proof. Set $w=-\min (u, 0)$. Then $u \in L^{2}\left[0, T ; H_{0}^{1,2}(\Omega)\right]$ and $u \geqslant 0$ on $\partial_{p} Q$ imply $w \in L^{2}\left[0, T ; H_{0}^{1,2}(\Omega)\right]$. Hence, a technique used to prove Lemma 1 in [1] can be used to show that the test function $v$ can be formally replaced with $w(x, t) \chi(s, t) \exp \{2 H(x, t)\}$ where

$$
H(x, t)=-\alpha|x-\xi|^{2} /(2 T-(t-s))-\beta(t-s)
$$

with $\alpha, \beta>0$ constants to be chosen, $\xi \in \Omega$ arbitrary and $s \in[0, T]$, and $\chi(s, t)$ the characteristic function. Then (7) becomes

$$
\begin{aligned}
0 \geqslant\left.\frac{1}{2} \int_{\Omega} w^{2} e^{2 H}\right|_{s} ^{t}+\int_{s}^{t} \int_{\Omega}\left[a_{i j} w_{, i} w_{, j}+2 a_{i j} H_{, j} w_{, i} w+d_{j} w_{, j} w\right. \\
\left.+2 d_{j} H_{, j} w^{2}-b_{j} w_{, j} w-c w^{2}-w^{2} H_{t}\right] e^{2 H} d x d t
\end{aligned}
$$

Since $H_{t}=-\left|H_{x}\right|^{2} / 4 \alpha-\beta$, choose $\alpha=\lambda\left(32 M^{2} n^{2}\right)^{-1}$ and set

$$
B(x, t)=\lambda^{-1} \sum\left(d_{j}-b_{j}\right)^{2}+\lambda(2 M n)^{-2} \sum d_{j}^{2}+c .
$$

Thus, $B \in L^{p, q}(Q)$ for some $(p, q)$ satisfying (*) and

$$
\int_{s}^{t} \int_{\Omega} B e^{2 H} w^{2} d x d t \geqslant-\|B\|_{p, q}\left\|e^{H} w\right\|_{2 p^{\prime}, 2 q^{\prime}}^{2}
$$

where the norms are taken over $\Omega \times(s, t)$. Then (8) becomes

$$
\begin{aligned}
0 \geqslant\left.\frac{1}{2} \int_{\Omega} e^{2 H} w^{2}\right|_{s} ^{t} d x+\frac{1}{2} \lambda\left\|e^{H} w_{x}\right\|_{2,2}^{2}+\beta\left\|e^{H} w\right\|_{2,2}^{2} \\
-\left(\|B\|_{p, q, Q}\right) K(t-s)^{\theta}\left\{\left\|e^{H} w\right\|_{2, \infty}^{2}+4\left\|e^{H} w_{x}\right\|_{2,2}^{2}\right. \\
\left.+\left[2 \alpha T^{-1} \operatorname{diam}(\Omega)\right]^{2}\left\|e^{H} w\right\|_{2,2}^{2}\right\} .
\end{aligned}
$$

Finally, by choosing $\beta=\left[2 \alpha T^{-1} \operatorname{diam}(\Omega)\right]^{2} K T^{\theta}\|B\|_{p, q, Q}$ and $\sigma$ so that

$$
K \sigma^{\theta}\|B\|_{p, q, Q} \leqslant \min (1 / 4, \lambda / 16),
$$

the inequality becomes

$$
\left.\int_{\Omega} e^{2 H} w^{2}\right|_{t} d x \leqslant\left.\int_{\Omega} e^{2 H} w^{2}\right|_{s} d x-\frac{1}{2} \lambda\left\|e^{H} w_{x}\right\|_{2,2}^{2}+\frac{1}{2}\left\|e^{H} w\right\|_{2, \infty}^{2} \cdot
$$


The right side is independent of $t$ in $(s, s+\sigma)$ and, hence,

$$
\frac{1}{2}\left\|e^{H} w\right\|_{2, \infty}^{2}+\frac{1}{2} \lambda\left\|e^{H} w_{x}\right\|_{2,2}^{2} \leqslant\left.\int_{\Omega} e^{2 H_{w}}\right|_{s} d x
$$

where the norms are taken over $\Omega \times(s, s+\sigma)$. Set $s=0$. Then $w(x, 0)=0$ implies

$$
\left\|e^{H} w\right\|_{2, \infty}^{2}+\left\|e^{H} w_{x}\right\|_{2,2}^{2}=0
$$

and, therefore, $w=0$ a.e. in $\Omega \times(0, \sigma)$. By iteration it follows that $w=0$ a.e. in $Q$.

COROllaRY. If $\iint_{Q}\left[c v-d_{j} v_{j}\right] d x d t \leqslant 0$ for all $v \in C_{0}^{1}(Q)$ with $v \geqslant 0$, then the solution $u$ of $L u=0$ in $Q, u=1$ on $\partial_{p} Q$ satisfies $0 \leqslant u(x, t) \leqslant 1$ in $Q$.

Henceforth, assume $c+\left\{d_{j}\right\}_{j} \leqslant 0$ weakly in $Q$. Then the representing measure $\mu_{(s, t)}$ obtained in Theorem 2 satisfies $0<a \leqslant \int d \mu_{(x, t)} \leqslant 1$ in $Q$ for some constant $a$.

IV. Generalized super-solutions. Let

$$
R_{a}\left(x_{0}, t_{0}\right)=\left\{(x, t) ;\left|x_{i}-x_{0 i}\right|<a, t_{0}-a^{2}<t<t_{0}\right\} \cup\left\{\left(x_{0}, t_{0}\right)\right\}
$$

denote a "parabolic rectangle" based at $\left(x_{0}, t_{0}\right)$ with "radius" a. For any such rectangle $R$ with $\bar{R} \subset Q$, the solution $u$ of the boundary value problem

$$
L u=0 \quad \text { in } R, \quad u=f \text { on } \partial_{p} Q,
$$

where $f$ is assumed continuous, satisfies

$$
u(z)=\int_{\partial_{p} R} f d \mu_{z} \equiv L(f ; z, R)
$$

for $z \in R$. Clearly, $L(f ; z, R)$ is defined on any rectangle $R$ with $\bar{R} \subset Q$ and $f \in C\left(\partial_{p} R\right)$. Hence, by the monotone convergence theorem, $L(f ; z, R)$ is defined for any lower semicontinuous function $f$ on $\partial_{p} R$. Therefore, if $f$ is semicontinuous on $\partial_{p} R$, then $f=f_{1}-f_{2}$ where $f_{1}$ and $f_{2}$ are nonnegative lower semicontinuous functions. Then

$$
L(f ; z, R)=L\left(f_{1} ; z, R\right)-L\left(f_{2} ; z, R\right)
$$

provided that, at each $z \in R$, at least one of the terms $L\left(f_{1} ; z, R\right), L\left(f_{2} ; z, R\right)$ is finite. This restriction will be included in the definition of generalized supersolutions.

DEFinition 4. Let $u$ be a Borel measurable function defined on an open set $D$. $u$ is

(a) locally super-mean-valued on $D$ (LSMV) if for each $z \in D$ there is rectangle $R_{z}$ with $\bar{R}_{z} \subset D$ and $z \in R_{z}$ such that, for each rectangle $R \subset R_{z}$ and 
each $y \in R, L(u ; y, R)$ is defined and $u(y) \geqslant L(u ; y, R)$.

(b) super-mean-valued on $D$ (SMV) if, for each $z \in D, L(u ; z, R)$ is defined and $u(z) \geqslant L(u ; z, R)$ for each rectangle $R$ with $z \in R$ and $\bar{R} \subset D$.

To get the corresponding definitions for sub-mean-valued functions the inequality is reversed. Then $u$ is mean-valued if it is both sub- and super-meanvalued.

Definition 5. Let $L_{Q}$ denote the class of extended real valued functions on $Q$ which satisfy

(i) $u \neq \equiv+\infty$ on $Q$,

(ii) $u>-\infty$ on $Q$,

(iii) $u$ is lower semicontinuous on $Q$.

Note that $L(u ; z, R)$ is defined on $R$ for each $u \in L_{Q}$ since $u$ is bounded below on $\partial_{p} R$.

Definition 6. (i) $S_{Q}^{\prime}=L_{Q} \cap\{u ; u$ is SMV $\}$.

(ii) $S_{Q}^{\prime \prime}=\left\{u \in L_{Q}\right.$; for each cylinder $W, \bar{W} \subset Q$, and each $v \in C(\bar{W})$ with $L v=0$ in $W$ and $v \leqslant u$ on $\partial_{p} W, v \leqslant u$ on $\left.W\right\}$.

(iii) $S_{Q}^{\prime \prime \prime}=L_{Q} \cap\{u ; u$ is LSMV $\}$.

One of the purposes of this paper is to prove $S_{Q} \subset S_{Q}^{\prime}=S_{Q}^{\prime \prime}=S_{Q}^{m}$.

The other purpose is to point out properties of functions which are in $S_{Q}^{\prime}$. Since $S_{Q} \subset S_{Q}^{\prime}$, functions in $S_{Q}^{\prime}$ are called generalized super-solutions of parabolic equations. It is obvious that $S_{Q} \subset S_{Q}^{\prime \prime}$. Before proving $S_{Q}^{\prime}=S_{Q}^{\prime \prime}=$ $S_{Q}^{m}$, a minimum principle is obtained.

THEOREM 4. If $u \in S_{Q}^{m}$ and if there is a $z_{0}=\left(x_{0}, t_{0}\right) \in Q$ such that $0 \geqslant$ $u\left(z_{0}\right)=\inf _{Q} u$, then $u(z)=u\left(z_{0}\right)$ on $\Omega \times\left(0, t_{0}\right)$.

Proof. Since $u \in S_{Q}^{m}$, there is a rectangle $P$ corresponding to $z_{0}$ with $\bar{P} \subset Q$ such that $u \in S_{P}^{\prime}$. Hence, for any $R \subset P$ with $z_{0} \in R, L\left(u ; z_{0}, R\right) \leqslant$ $u\left(z_{0}\right) \leqslant 0$ and, therefore, $\inf _{\partial_{p} R} u \leqslant 0$. It follows that

$$
L\left(u ; z_{0}, R\right) \geqslant \inf _{\partial_{p} R} u \cdot L\left(1 ; z_{0}, R\right) \geqslant \inf _{\partial_{p} R} u \geqslant u\left(z_{0}\right) .
$$

Thus, for any rectangle $R$ with $z_{0} \in R$ and $R \subset P, u\left(z_{0}\right)=L\left(u ; z_{0}, R\right)$.

Set $Q_{0}=\left\{\Omega \times\left(0, t_{0}\right)\right\} \cup\left\{\left(x_{0}, t_{0}\right)\right\}$ and let $M=\left\{z \in Q_{0} ; u(z)=u\left(z_{0}\right)\right\}$. Clearly $M \neq \varnothing$. Since $u$ is lower semicontinuous, $M$ is relatively closed. To see that $M$ is relatively open, let $y \in M$. Since $y$ is LSMV on $Q$, there is a rectangle $R_{y}, \bar{R}_{y} \subset Q$, such that $u(y) \geqslant L(u ; y, R)$ for every $R, R \subset R_{y}$, and $y \in R$. If it can be shown that $R_{y} \subset M$, then $M$ will be relatively open in $Q_{0}$ and, hence, $Q_{0}=M$.

For contradiction assume there is a $z \in R_{y}-M$ and let $R$ be a rectangle with $y \in R, z \in \partial_{p} R$, and $\bar{R} \subset R_{y}$. Then, since $y \in M, u(y)=L(u ; y, R)$ and, hence, 


$$
\begin{aligned}
0 & \leqslant L(u-u(y) ; y, R)=L(u ; y, R)-u(y) L(1 ; z, R) \\
& =u(y)[1-L(1 ; y, R)] \leqslant 0 .
\end{aligned}
$$

Thus, $L(u-u(y) ; y, R)=0$ and $u=u(y) \mu_{y}$-almost everywhere on $\partial_{p} R$.

Since $z \in \partial_{p} R \cap\left(R_{a}-M\right), u(z)>u(y)$ and, for any $\alpha$ with $u(z)>\alpha>$ $u(y)$, the lower semicontinuity of $u$ implies there is a neighborhood $U_{z, \alpha}$ about $z$ such that $u>\alpha$ in $U_{z, \alpha}$. Then

$$
0=L(u-u(y) ; z, R) \geqslant \int_{U_{z, \alpha}}(u-u(y)) d \mu_{y} \geqslant[\alpha-u(y)] \mu_{y}\left(U_{z, \alpha}\right) .
$$

Since $u(y)<\alpha, \mu_{y}$ has zero support on $\partial_{p} R \cap U_{z, \alpha} \equiv O_{z, \alpha}$, an open subset of $\partial_{p} R$. This will be shown to be impossible and, therefore, no such $z$ can exist.

Let $C_{z, \alpha} \subset O_{z, \alpha}$ be closed in $\partial_{p} R$ and have positive measure. Let $f \in$ $C\left(\partial_{p} R\right)$ with $f \equiv 0$ on $\partial_{p} R-O_{z, \alpha} f \geqslant 0$, and $f \equiv 1$ on $C_{z, \alpha}$. Then the solution $v$ of the boundary value problem

$$
L v=0 \text { in } R, \quad v=f \text { on } \partial_{p} R
$$

satisfies $v(z)=L(f ; z, R)=0$ in $R$. However, Trudinger has shown [8] that $v \in C(\bar{R})$. This is impossible since $f \equiv 1$ on $C_{z, \alpha}$.

THEOREM 5. $S_{Q}^{\prime}=S_{Q}^{\prime \prime}=S_{Q}^{m}$.

Proof. It is obvious that $S_{Q}^{\prime} \subset S_{Q}^{m}$. Let $u \in S_{Q}^{m}$. To see that $u \in S_{Q}^{\prime \prime}$, let $W$ be a cylinder with $\bar{W} \subset Q$. Let $v \in C(\bar{W})$ with $L v=0$ in $W$ and $v \leqslant u$ on $\partial_{p} W$. Set $w=u-v$. Then $w$ is LSMV on $W$. If $u<v$ somewhere on $W$, then there is a $z_{0} \in W$ such that $w\left(z_{0}\right)=\inf _{W} w<0$. Hence, by the minimum principle $w=w\left(z_{0}\right)<0$ in $W \cap\left(0, t_{0}\right)$. This is impossible since $w \geqslant 0$ on $\partial_{p} W$. Hence $w \geqslant 0$ and $u \in S_{Q}^{\prime \prime}$.

Next assume $u \in S_{Q}^{n}$; it must be shown that $u$ is SMV. Let $R$ be a rectangle with $\bar{R} \subset Q$. Since $u$ is lower semicontinuous on $\bar{R}$, let $\left\{\phi_{j}\right\} \subset C(\bar{R})$ be such that $\phi_{j} \uparrow u$. Let $v_{j}$ be the solution of the boundary value problem

$$
L v=0 \quad \text { in } R, \quad v=\phi_{j} \quad \text { on } \partial_{p} R .
$$

Then, by [8], $v_{j} \in C(\bar{R})$ and, since $L v_{j}=0$ in $R$ and $v_{j}=\phi_{j} \leqslant u$ on $\partial_{p} R, u \in$ $S_{Q}^{n}$ implies $v_{j} \leqslant u$ in $R$. Therefore,

$$
u(z) \geqslant v_{j}(z)=L\left(\phi_{j} ; z, R\right) \text { in } R .
$$

It follows from the monotone convergence theorem that

$$
u(z) \geqslant L(u ; z, R) \text {. }
$$

V. Properties of generalized super-solutions. Before showing some properties of super-solutions, a few examples are given. 
A. Let $u$ satisfy $L u=0$ in $Q$. Then $u,-u$, and $-|u| \in S_{Q}^{\prime}$.

B. Let $\Gamma(x, t ; \xi, \tau)$ denote the fundamental solution of $L u=0$ in $E^{n} x(-1,2 T)$ whose existence was shown in [1]. For fixed $(\xi, \tau)$ set

$$
G(x, t ; \xi, \tau)= \begin{cases}\Gamma(x, t ; \xi, \tau), & t>\tau, \\ 0, & t \leqslant \tau .\end{cases}
$$

Then $G(x, t ; \xi, \tau) \in S_{Q}^{\prime}$.

C. If $u$ satisfies $L u=0$ in $Q$, then $u^{+} \equiv u \vee O \in S_{Q}^{\prime}$.

A class of super-solutions can be obtained as follows:

THEOREM 6. Let $C(x)$ be a convex function on $E^{1}$ with $C(0) \leqslant 0$. If $u$ satisfies $L u=0$ in $Q$, then $-C(u) \in S_{Q}^{\prime}$.

Proof. Clearly, $-C(u) \in L_{Q}$. Therefore, it is only necessary to show $C(u)(z) \leqslant L(C(u) ; z, R)$ for any rectangle $R, \bar{R} \subset Q$, and any $z \in R$. Since $u(z)$ $=L(u ; z, R)$,

$$
C(u)(z)=C(L(1 ; z, R) \cdot L(u ; z, R) / L(1 ; z, R))
$$

provided $L(1 ; z, R)>0$. If, however, $L(1 ; z, R)=0$, then $\mu_{z}\left(\partial_{p} R\right)=0$, and, since $u(z) \equiv 0$,

$$
C(u)(z)=C(0) \leqslant 0=L(C(u) ; z, R) .
$$

Therefore, assume $0<L(1 ; z, R) \leqslant 1$ on $R$. Since $C$ is convex,

$$
\begin{aligned}
C(u)(z) & \leqslant L(1 ; z, R) C(L(u ; z, R) / L(1 ; z, R))+[1-L(1 ; z, R)] C(0) \\
& \leqslant L(1 ; z, R) C(L(u ; z, R) / L(1 ; z, R)) .
\end{aligned}
$$

It follows from Jensen's inequality that

$$
C(L(u ; z, R) / L(1 ; z, R)) \leqslant L(C(u) ; z, R) / L(1 ; z, R) .
$$

Therefore, $C(u)(z) \leqslant L(C(u) ; z, R)$.

COROLlaRY. If $C(x)$ is a nondecreasing convex function with $C(0) \leqslant 0$ and if $-u \in S_{Q}^{\prime}$, then $-C(u) \in S_{Q}^{\prime}$.

It follows from Example $A$ and this corollary that $-u \in S_{Q}^{\prime}$ implies $-|u|^{p} \in S_{Q}^{\prime}$ provided $p \geqslant 1$.

Before showing that a sum of functions in $S_{Q}^{\prime}$ is in $S_{Q}^{\prime}$ it is necessary to prove the following lemma.

LemmA 1. Let $u \in S_{Q}^{\prime}$. If $E \subset Q$ is such that $E \cap\left(t_{0}\right)$ has positive $n$ dimensional measure for some $t_{0} \in(0, T)$ and if $u \equiv+\infty$ on $E$, then $u \equiv+\infty$ on $\Omega \times\left(t_{0}, T\right)$. 
Proof. Let $R_{a}(x, t)$ be any standard rectangle in $Q$ such that $\partial_{p} R_{a} \cap$ $E \cap\left(t_{0}\right)$ has positive $n$-dimensional measure. Let $z=(y, s)$ be any point in $R_{a}$. It follows from the representation of solutions given in [1, Theorem 9] that for a measurable set $A \subset \partial_{p} R_{a} \cap\left(t-a^{2}\right)$,

$$
\mu_{z}(A)=\int_{\{|\xi-x|<a\}} \chi_{A}(\xi) \gamma_{a}\left(y, s ; \xi, t-a^{2}\right) d \xi
$$

where $\gamma_{a}(y, s ; \xi, \tau)$ is the Green's function for $L u=0$ in $R_{a}(x, t)$. Then $\mu_{z} \geqslant 0$ and $u \in S_{Q}^{\prime}$ imply

$$
u(z) \geqslant L\left(u ; z, R_{a}\right) \geqslant \int_{\{|\xi-x|<a\}} u\left(\xi, t-a^{2}\right) \gamma_{a}\left(y, s ; \xi, t-a^{2}\right) d \xi .
$$

Since $\gamma_{a}$ is bounded below by a positive constant on each subset of $\{|\xi-x|<$ a) $\times\left(t-a^{2}\right)$ for fixed $(y, s) \in R_{a}(x, t)$, it follows that this last integral equals $+\infty$.

It follows from Lemma 1 that $u \in S_{Q}^{\prime}$ implies $u$ must satisfy exactly one of the following:

(i) $u \equiv+\infty$ on $Q$,

(ii) there is a $t_{0} \in(0, T)$ such that $u<+\infty$ on $\Omega \times\left(0, t_{0}\right)$ and $u \equiv$ $+\infty$ on $\Omega \times\left(t_{0}, T\right)$

(iii) $u<\infty$ on $Q$.

However, $u \in S_{Q}^{\prime}$ implies $u \in L_{Q}$ and (i) is impossible. Therefore, the following corollary can be stated.

CoRollary. If $u \in S_{Q}^{\prime}$, there is a $t_{0} \in(0, T]$ such that $u<\infty$ on $\Omega \times$ $\left(0, t_{0}\right)$ and $u \equiv+\infty$ on $\Omega \times\left(t_{0}, T\right)$.

THEOREM 7. If $u, v \in S_{Q}^{\prime}$ and $c>0$, then $c u, u \wedge v$, and $u+v \in S_{Q}^{\prime}$.

Proof. It is obvious that $c u \in S_{Q}^{\prime}$. To see that $u \wedge v \in S_{Q}^{\prime}$, the lower semicontinuity follows from $\{z ; u \wedge v(z)>\alpha\}=\{z ; u(z)>\alpha\} \cap\{z ; v(z)>\alpha\}$ and the super-mean-value property is trivial to show. Finally, to see that $u+$ $v \in S_{Q}^{\prime}$, the corollary to Theorem 6 implies that $u+v \in L_{Q}$ since $u, v \in L_{Q}$. Since the super-mean-value property is obvious, $u+v \in S_{Q}^{\prime}$.

TheOREM 8. $u,-u \in S_{Q}^{\prime}$ imply $u$ is a weak solution of $L u=0$ in $Q$.

Note. The strength of this theorem lies in the fact that no differentiability assumptions are explicitly made.

Proof. Since $u \in C(Q)$, let $R$ be a standard rectangle in $Q$ and let $v$ satisfy

$$
L v=0 \text { in } R, \quad v=u \text { on } \partial_{p} R .
$$

Then, since $u,-u \in S_{Q}^{\prime}$ imply $u(z)=L(u ; z, R), v(z)=L(u ; z, R)=u(z)$ in $R$. Since $v$ satisfies $L v=0$ in $R, u$ does also. Since $R \subset Q$ was arbitrary, $L u=0$ in $Q$. 
THEOREM 9. If $u \in S_{Q}^{\prime}$, then $u(z)=\liminf _{y \rightarrow z ; y \neq z} u(y)$.

Proof. The inequality $\leqslant$ follows from the lower semicontinuity of $u$. If $u(z)=+\infty$, the proof is complete. Thus, assume $u(z)<\infty$. Then

$$
u(z) \geqslant L(u ; z, R) \geqslant \inf _{\partial_{p} R} u \cdot L(1 ; z, R) .
$$

The desired result will follow if it can be shown that, for arbitrary $\epsilon>0,1-$ $\epsilon<L(1 ; z, R) \leqslant 1$ for sufficiently small rectangles $R$. However, this follows from $L(1 ; z, R) \leqslant 1$ and from the maximum principle in [2].

THEOREM 10. Let $u \in S_{Q}^{\prime}$ and let $R$ be a standard rectangle in $Q$. If $u<\infty$ on $R$, then $L(u ; z, R)$ exists and the function

$$
v(z)= \begin{cases}L(u ; z, R), & z \in R, \\ u(z), & z \in Q-R,\end{cases}
$$

satisfies $u \geqslant v, L v=0$ on $R$, and $v \in S_{Q}^{\prime}$.

PRoof. The existence of $L(u ; z, R)$ is obtained using the fact that $u \in$ $L_{Q}$. Let $w_{j} \in C(Q)$ with $w_{j} \uparrow u$. Let $v_{j}$ satisfy $L v_{j}=0$ in $R, v_{j}=w_{j}$ on $\partial_{p} R$. Since $v_{j} \leqslant u$ on $\partial_{p} R, u \in S_{Q}^{\prime}$ implies $v_{j} \leqslant u$ on $R$. Hence, $u(z) \geqslant v_{j}(z)=$ $L\left(w_{j} ; z, R\right)$ on $R$. Since $u<\infty$ on $R$, the monotone convergence theorem implies that $u(z) \geqslant L(u ; z, R)$. This shows that $L(u ; z, R)$ exists and $v(z) \leqslant u(z)$ on $Q$.

Since $v_{j} \in C(\bar{R})$ and $L v_{j}=0$ in $R$, an argument in the proof of Theorem 1 can be repeated to show that $v$ satisfies $L v=0$ in $R$. To see that $v \in S_{Q}^{m}$, let $z_{0} \in \partial_{p} R$. For any rectangle $R_{1}$ with $z_{0} \in R_{1}$ and $\bar{R}_{1} \subset Q$, it is clear that

$$
v\left(z_{0}\right)=u\left(z_{0}\right) \geqslant L\left(u ; z_{0}, R\right) \geqslant L\left(v ; z_{0}, R\right) .
$$

The lower semicontinuity of $v$ follows from the fact that

$$
V_{j}(z)= \begin{cases}v_{j}(z), & z \in R, \\ w_{j}(z), & z \in Q-R,\end{cases}
$$

satisfies $V_{j} \in C(Q)$-from [8]-and $V_{j} \uparrow v$ on $Q$.

\section{REFERENCES}

7. Neil A. Eklund, Existence and representation of solutions of parabolic equations, Proc. Amer. Math. Soc. 47 (1975), 137-142.

8. Neil Trudinger, Pointwise estimates and quasilinear parabolic equations, Comm. Pure Appl. Math. 21 (1968), 206-226. MR 37 \#1758.

DEPARTMENT OF MATHEMATICS, CENTRE COLLEGE OF KENTUCKY, DANVILLE, KENTUCKY 40422 